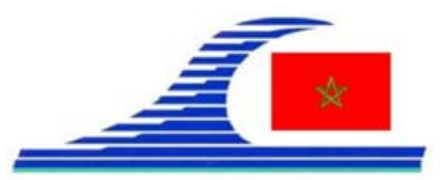

Conférence Méditerranéenne Côtière et Maritime

EDITION 2, TANGER, MAROC (2011)

Coastal and Maritime Mediterranean Conference

Disponible en ligne - http://www.paralia.fr - Available online

\title{
Etude, en zone méditerranéenne, de l'influence des aérosols marins côtiers sur les comportements microphysiques des nuages convectifs
}

\author{
Rabia MERROUCHI ${ }^{\mathbf{1}}$, Jacques PIAZZOLA ${ }^{2}$, \\ Mohamed CHAGDALI ${ }^{3}$, Soumia MORDANE ${ }^{3}$
}

1. Direction de la Météorologie Nationale, Casablanca, Maroc. rabia.merrouchi@gmail.com

2. ISITV, Laboratoire des Echanges Particulaires aux Interfaces (LEPI), Université de Toulon Var, France.

jacques.piazzola@univ-tln.fr

3. Laboratoire de Calcul Scientifique en Mécanique, Faculté des sciences Ben M’sick

Casablanca, Maroc.

m_chagdalli@hotmail.com et mordanesoumia@yahoo.fr

\section{Résumé :}

L'interaction aérosol nuage est un mécanisme fondamental qui influence les comportements microphysiques des nuages et en particulier le processus de précipitation. Etant donné que la mer constitue la première source des aérosols atmosphériques naturels et afin d'étudier l'impact des aérosols marins, et en particulier les aérosols marins côtiers, sur le comportement microphysique des nuages, des simulations ont été conduites utilisant un modèle de nuage à microphysique détaillée et an ayant recours à différents spectres d'aérosols marins dont des spectres expérimentaux mesurés en méditerranée.

La prise en considération de la production naturelle des aérosols marins côtiers a induit une nette modification dans certaines propriétés microphysiques du nuage par rapport aux résultats obtenus en utilisant les autres spectres d'aérosol continental ou marins. De telles modifications dans les propriétés microphysiques du nuage ont une influence directe sur les conditions de déclanchement des précipitations ainsi que sur les quantités recueillies au sol.

\section{Mots-clés :}

Spectre d'aérosols marins côtiers - Modèle de nuage - Microphysique des nuages Précipitations.

\section{Introduction}

L'interaction aérosol nuage est un mécanisme fondamental influençant le processus de précipitation. En effet, les processus microphysiques régissant la croissance des gouttelettes d'eau à l'intérieur du nuage dépendent du spectre initial et de la 
La connaissance de la Mer :

un vecteur du développement durable en Méditerranée

composition chimique des aérosols atmosphériques pris dans les courants ascendants alimentant ce dernier.

La nécessité d’introduire un terme source pertinent dans les modèles numériques de nuages et de transport atmosphérique explique l'intérêt que soulève depuis plusieurs années l'étude des processus de génération de l'aérosol produit par le déferlement des vagues. La méthodologie, adoptée pour ce travail, est basée sur l'étude des relations entre le taux de couverture moutonneuse (witecapping) et les paramètres météorologiques (VIGNATI et al., 2001 ; PIAZZOLA et al., 2002) couplées à des mesures de surface de déferlement (PIAZZOLA et al., 2003).

Afin d'étudier l'impact des aérosols marins sur le comportement microphysique des nuages et en particulier celui des spectres marins côtiers produits en méditerranée, des simulations numériques ont été conduites reliant le modèle (MEDEX) de production des aérosols marins côtiers au modèle de nuage à microphysique détaillée (ExMIX).

Ces simulations ont porté sur un nuage convectif (à phase mixte) en utilisant des spectres initiaux d'aérosols marins d'origines différentes.

\section{Méthodes}

\subsection{Le modèle MEDEX :}

Le modèle MEDEX calcule la distribution de taille des particules d'aérosols marins. MEDEX a été développé sur la base d'une série de mesures acquises sur l'île de Porquerolles (Toulon- France) entre 2000 et 2001. Durant cette période, une large variété de distribution d'aérosols a été enregistrée sous différentes conditions météorologiques. La distribution des particules est prise comme étant la somme de quatre fonctions log- normales dont les modes sont paramétrés en fonction de la vitesse du vent et de la longueur du fetch. Les résultats de ce modèle ont été comparés à d'autres observations effectuées en mer noire et en mer méditerranée (PIAZZOLA \& KALOSHIN, 2005).

\subsection{Le modèle de nuage ExMIX}

Le modèle $1 \mathrm{D}^{1} \frac{1}{2}$ de nuage EXMIX (EXternally MIXture) a été développé par le Laboratoire de Météorologie Physique (MONIER et al., 2006 ; LEROY et al., 2006) se basant sur un concept de suivi pas à pas de l'évolution du spectre des particules d'aérosol (humidification puis formation et croissance des gouttes et cristaux de glace). Différents processus microphysiques de formation et de croissance des particules humides et solides sont pris en considération dans le modèle.

\subsection{Les Simulations}

Les simulations conduites ont porté principalement sur la modification du spectre initial d’aérosols comme illustré dans le tableau 1. 
A best knowledge of the Sea: A sustainable development vector in Mediterranean

Tableau 1. Synthèse des paramètres caractéristiques des spectres d'aérosols utilisés.

\begin{tabular}{|c|c|c|c|}
\hline Spectre & Concentration $N_{i}$ & Rayon $R_{i}$ & $\log \left(\sigma_{i}\right)$ \\
\hline Continental à trois modes & $N_{l}=997 \mathrm{~cm}^{-3}$ & $R_{1}=0.001 \mu \mathrm{m}$ & 0.328 \\
\hline \multirow{2}{*}{$\begin{array}{l}\text { lognormaux observé au puy du } \\
\text { dôme-France. SELLEGRI (2002) }\end{array}$} & $N_{2}=842 \mathrm{~cm}^{-3}$ & $R_{2}=0.0218 \mu \mathrm{m}$ & 0.505 \\
\hline & $N_{3}=7.1 \cdot 10^{-4} \mathrm{~cm}^{-3}$ & $R_{3}=6.24 \mu \mathrm{m}$ & 0.277 \\
\hline \multirow{3}{*}{$\begin{array}{l}\text { Océanique à trois modes } \\
\text { lognormaux-JAENICKE (1988) }\end{array}$} & $N_{l}=133 \mathrm{~cm}^{-3}$ & $R_{1}=0.004 \mu m$ & 0.66 \\
\hline & $N_{2}=66,6 \mathrm{~cm}^{-3}$ & $R_{2}=0.133 \mu \mathrm{m}$ & 0.21 \\
\hline & $N_{3}=3.1 \mathrm{~cm}^{-3}$ & $R_{3}=0.290 \mu \mathrm{m}$ & 0.40 \\
\hline \multirow{4}{*}{$\begin{array}{l}\text { Marin côtier issu de Medex pour un } \\
\text { vent } U=20 \mathrm{~m} / \mathrm{s} \text { et un fetch } X=30 \mathrm{~km} \text { et } \\
R H=80 . \text { PIAZOLA et al. }\end{array}$} & $A_{1} / f=92634,22 \mathrm{~cm}^{-3}$ & $R_{l}=0.03 \mu \mathrm{m}$ & $C_{1}=1,241$ \\
\hline & $A_{2} / f=55,713 \mathrm{~cm}^{-3}$ & $R_{2}=0.24 \mu \mathrm{m}$ & $C_{2}=1,357$ \\
\hline & $A_{3} / f=0,449 \mathrm{~cm}^{-3}$ & $R_{3}=2.0 \mu \mathrm{m}$ & $C_{3}=1,508$ \\
\hline & $A_{4} / f=0,008 \mathrm{~cm}^{-3}$ & $R_{4}=10.0 \mu \mathrm{m}$ & $C_{4}=10$ \\
\hline \multirow{3}{*}{$\begin{array}{l}\text { Marin d'après VIGNATI et al. } \\
\text { (2001) } \\
\text { Pour un vent } U=20 \mathrm{~m} / \mathrm{s} \text { et } R H=80 \%\end{array}$} & $N_{l}=152,40 \mathrm{~cm}^{-3}$ & $R_{l}=0.2 \mu m$ & 0.278 \\
\hline & $N_{2}=13,55 \mathrm{~cm}^{-3}$ & $R_{2}=2.0 \mu \mathrm{m}$ & 0.301 \\
\hline & $N_{3}=0,0075 \mathrm{~cm}^{-3}$ & $R_{3}=12 \mu \mathrm{m}$ & 0.477 \\
\hline
\end{tabular}

\section{Résultats et Conclusions:}

Dans cette étude, différents types de spectre d'aérosols marins ont été utilisés. Le passage d'un spectre d'aérosol continental à un spectre d'aérosols marins est synonyme de l'introduction de particules d'aérosols de grande taille. L'impact d'un tel changement dans le spectre initial des aérosols est senti en termes de réponses dynamiques et microphysiques des nuages favorisant un déclenchement précoce du processus de précipitation et un accroissement des quantités de précipitations recueillies au sol. De tels changements sont encore plus importants en présence d'aérosols marins produits naturellement en zone côtière (spectre MEDEX en méditerranée).

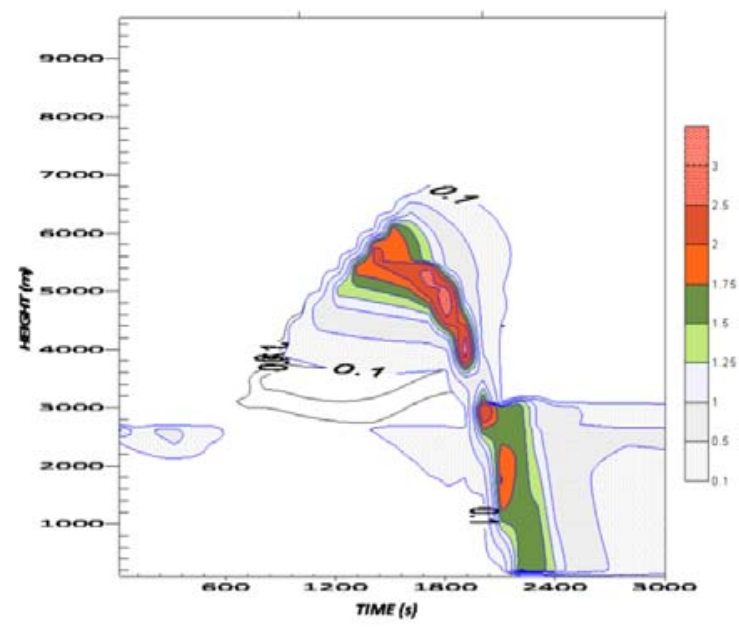

Figure 1.a. Évolution du Contenu en eau en $\mathrm{g} / \mathrm{m}^{3}$. Spectre MEDEX.

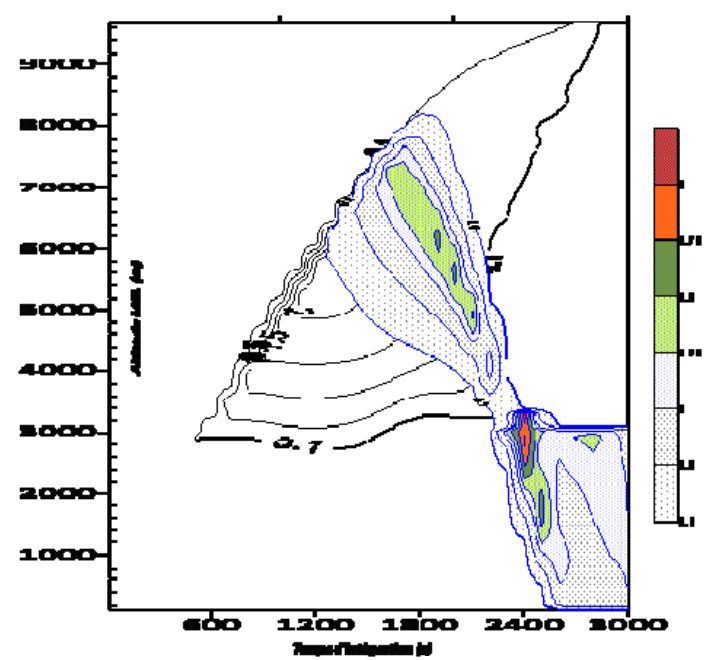

Figure 1.b. Évolution du Contenu en eau liquide en $\mathrm{g} / \mathrm{m}^{3}$. Spectre de Jaenicke. 
La connaissance de la Mer :

un vecteur du développement durable en Méditerranée

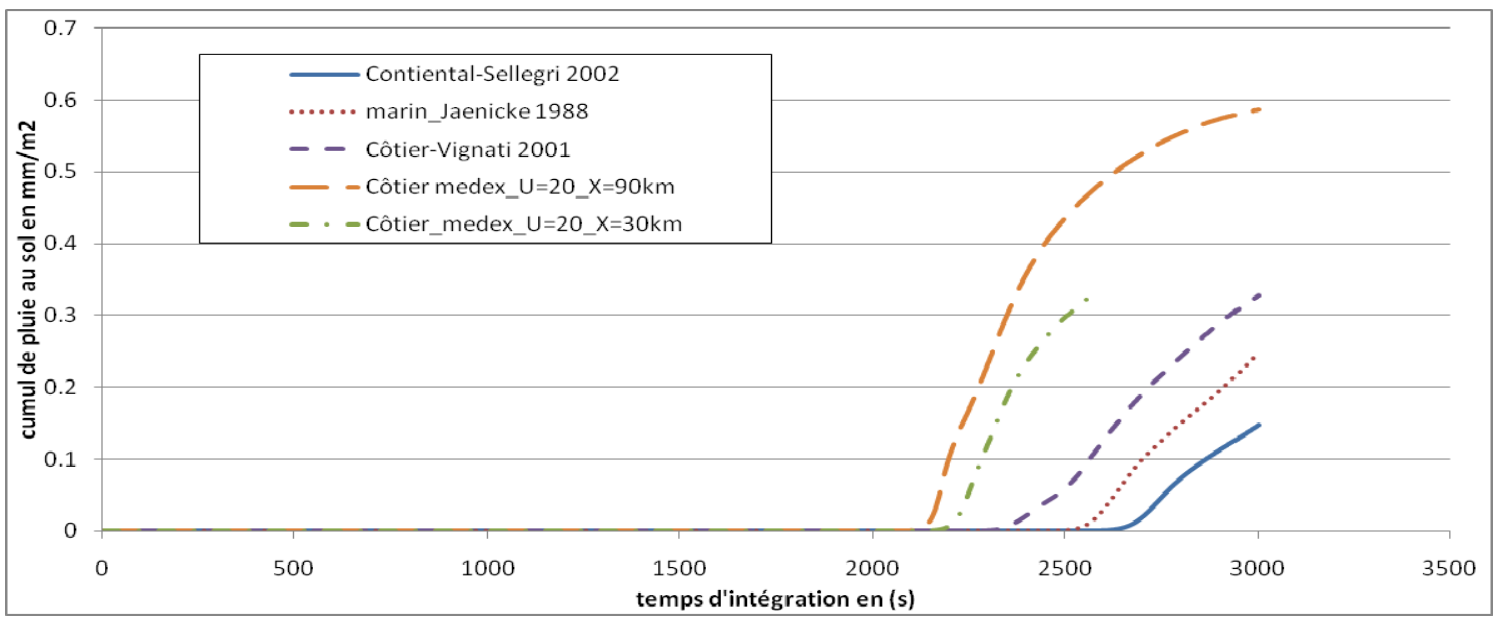

Figure 2. Évolution du cumul de pluie recueillie au sol pour les différentes simulations. $U$ désigne la vitesse du vent à $10 \mathrm{~m}$ et X la longueur du fetch.

\section{Références bibliographiques}

JAENICKE R. (1988). Aerosol physics and chemistry. In: Landolt-Boernstein: Zahlenwerte und Funktionen aus Naturwissenschaften und Tecknik, V 4b, G. Fischer, Editor, Springer, pp 391-457.

LEROY D., MONIER M., WOBROCK W., FLOSSMANN A.I. (2006). A numerical study of the effects of the aerosol particle spectrum on the development of the ice phase and precipitation formation. Atmos. Res., 80: pp 15-45. doi:10.1016/j.atmosres.2005.06.007 MONIER M., WORBROCK W., GAYET J.-F, FLOSSMANN A. (2006). development of a detailed microphysics cirrus model tracking aerosol particles' histories for interpretation of the recent INCA campaign. Journal of the Atmospheric Sciences 63. pp 504-525. doi:10.1175/JAS3656.1

PIAZZOLA J., KALOSHIN G. (2005). Performance evaluation of the coastal aerosol extinction code MEDEX with data from the Black Sea. Journal of Aerosol Science, 36, pp 341-359. doi:10.1016/j.jaerosci.2004.09.009

PIAZZOLA J., BOUCHARA F., VAN EIJK A.M.J., DE LEEUW G. (2003). Development of the Mediterranean extinction code MEDEX, Optical Engineering, Vol. 42, n 4 , pp 912-924. doi:10.1117/1.1556765

PIAZZOLA J., FORGET P., DESPIAU S. (2002). A Sea spray generation function for fetch-limited conditions. Annales Geophysicae, 20, pp 121-131. doi:10.5194/angeo-20-1212002

SELLEGRI K. (2002). Etude du processus d'activation des particules d'aérosols : implications en chimie multiphases. Thèse de l’Université Joseph Fourier, Grenoble.

VIGNATI E., DE LEEUW G., BERKOWICZ R. (2001). Modeling coastal aerosol transport and effects of surf-produced aerosols on processes in the marine atmospheric boundary layer, J. Geophys. Res., 106(D17), pp 20,225-20,238. doi:10.1029/2000JD000025 